\title{
EFECTO DE LAS CONDICIONES DE ALMACENAMIENTO EN LA DEGRADACIÓN DE CIPERMETRINA EN PASTOS EN CLIMA FRIO MODERADO
}

\author{
Yaneth Cardona1 „, Manuel Peláez²., Amanda Chaparro³ \\ 1. Departamento de Química, Universidad de Pamplona. \\ ${ }^{2}$ Departamento de Ciencias Biológicas. Universidad Nacional de Colombia-Sede Palmira, Colombia. \\ 3 Departamento de Química, Universidad de Pamplona.
}

\section{Resumen}

Para mantener la integridad de las muestras durante las determinaciones analíticas, es importante controlar la manipulación y preservación de las mismas, pues no es común realizar los análisis in situ; por lo cual, las muestras deben ser transportadas a un laboratorio antes de su análisis. Diferentes factores contribuyen a la inestabilidad de los pesticidas piretroides, como la composición de la matriz, material del recipiente de almacenamiento, acidez de la muestra, entre otros. El objetivo de este trabajo fue determinar las condiciones adecuadas de almacenamiento de tres tipos de pasto (Pennisetum clandestinum, Brachiaria decumbens y Cynodon nlemfuensis), que garanticen la integridad de la cipermetrina en estas muestras. Se compararon cuatro tipos de recipientes y ocho condiciones diferentes de almacenamiento, utilizando microextracción en fase sólida (SPME) y cromatografía de gases con detector de ionización de llama (GCFID) como técnicas de extracción y determinación, para estudiar la degradación de cipermetrina. Se encontró que las muestras de forraje estudiadas se pueden almacenar durante cinco días previos al análisis, en bolsas de polietileno de alta densidad o aluminio, en la nevera a una temperatura entre $5^{\circ} \mathrm{C}$ y $-4^{\circ} \mathrm{C}$.

Palabras clave: Cipermetrina, degradación, pesticidas, SPME, GC-FID

\section{EFFECT OF STORAGE CONDITIONS IN DEGRADATION OF CYPERMETHRIN IN PASTURES IN MODERATE COLD WEATHER}

\begin{abstract}
To maintain the integrity of the samples for the analysis, it is important to control the handling and preservation of them, because it is not common to perform determinations in situ; therefore, the samples must be transported to a laboratory before analyzes. Different factors contribute to the instability of pyrethroid pesticides, such as the composition of the matrix, material of the storage container, acidity of the sample, among others. The goal of this study was to determine appropriate storage condition in three types of grass (Pennisetum clandestinum, Brachiaria decumbens y Cynodon nlemfuensis) to ensure the integrity of cypermethrin in these samples. Four types of containers and eight different storage conditions were compared, using solid phase micro extraction
\end{abstract}


(SPME) and gas chromatography with flame ionization detector (GC-FID) as extraction and quantification techniques, to study cypermethrin degradation. It was found that the forage samples studied can be stored for five days prior to analysis, in high density polyethylene or aluminum bags, in the refrigerator at temperatures between $5^{\circ} \mathrm{C}$ and $-4^{\circ} \mathrm{C}$.

Keywords: Cypermethrin, degradation ,pesticides, SPME, GC-FID.

\footnotetext{
*Para citar este artículo: Cardona Y., Peláez M., Chaparro A. Efecto de las condiciones de almacenamiento en la degradación de cipermetrina en pastos en clima frio moderado. Revista Bistua. 2015.13(2):29-36
}

+ Autor para el envió de correspondencia y la solicitud de las separatas: Chaparro A. Universidad de Pamplona. Facultad de Ciencias Basicas.Departamento de Química. email:achaparro@unipamplona.edu.co

\section{INTRODUCCIÓN}

La cipermetrina es un pesticida piretroide, sintetizado en 1974 e introducido al mercado en 1977; está clasificado por la Organización Mundial de la Salud, como "moderadamente peligrosa" (clase II); sin embargo, algunos estudios muestran que los efectos de la cipermetrina y de los piretroides en general en la salud pueden ser más severos de lo que fue sugerido en evaluaciones toxicológicas previas. Este pesticida está registrado a nivel mundial para manejo de insectosplaga en pastos (Collaria scenica), cosechas, invernáculos, campos 
agrícolas y tratamiento de postcosecha ${ }^{1,2}$.

Actualmente, la cipermetrina es uno de los insecticidas más utilizados en la práctica de la siembra directa; dentro de los cultivos con mayor demanda en el país se encuentran los pastos. Las praderas que son utilizadas para la alimentación animal ocupan el tercer renglón en la aplicación de plaguicidas. El uso de este piretroide a nivel mundial incrementó drásticamente en múltiples aplicaciones debido a su eficacia en pequeñas dosis, su alta proporción de degradación en el ambiente y su toxicidad relativamente baja con respecto a los organismos no blancos 3-6.

Estudios realizados indican que luego de que el insecticida es aplicado sobre los cultivos, sus residuos pueden ser encontrados en suelos, aguas superficiales y sedimentos; sin embargo, la degradación biológica es relativamente rápida y los residuos no permanecen de manera prolongada en el ambiente. La fotodegradación y la biodegradación, por ejemplo, son procesos que pueden limpiar naturalmente al ambiente. La sensibilidad del pesticida a la luz y a ciertas temperaturas son los dos

factores más importantes que determinan la duración residual de estos productos, por lo cual se han evaluado los efectos de almacenamiento en la degradación de algunos pesticidas, en diversas matrices como suelo, agua, frutas, entre otros, con fines de inspección y vigilancia de la normatividad correspondiente (límites de MRL) y para impulsar la creación de nuevas legislaciones en aquellas matrices que carecen de la misma. Sin embargo, en ocasiones es tan grande la cantidad de muestras a analizar que se hace necesario un método de almacenamiento de las mismas, que garantice la menor velocidad de degradación posible del analito en las muestras para la obtención de resultados confiables ${ }^{7-12}$.

Por lo tanto en este trabajo se evaluó la vida útil de cipermetrina en 3 tipos de pasto (Pennisetum clandestinum, Cynodon nlemfuensis y Brachiaria decumbens) de uso común en agroecosistemas de pastura de trópico alto, medio y bajo respectivamente, para determinar las condiciones de almacenamiento adecuadas, previas a su análisis, en un laboratorio de clima frío-moderado, como el laboratorio de control de calidad de la Universidad de Pamplona (2350msnm, $16^{\circ} \mathrm{C}, 80 \mathrm{HR}$ ) y asegurar la cuantificación de estos contaminantes mediante microextracción en fase sólida (SPME) y cromatografía de gases con detector de ionización de llama (GC-FID).

\section{METODOLOGIA}

Los ensayos se realizaron por duplicado en el laboratorio de control de calidad de la universidad de Pamplona.

\section{Materiales y Reactivos}

Las muestras de pasto fueron dopadas con estándar analítico cipermetrina (mezcla de isómeros, Fluka) 95,1\% y acetona (grado de pureza HPLC, Merck).

Las extracciones y los análisis cromatográficos se llevaron a cabo utilizando un soporte manual para SPME (Supelco), una fibra de Polidimetilsiloxano-Divinilbenceno

(PDMS-DVB) con grosor de $65 \mu \mathrm{m}$ (acondicionada antes de su uso siguiendo las recomendaciones del fabricante) y un cromatógrafo de gases Agilent 6890 con puerto de inyección Split-splitless y detector de 
Ionización de llama (FID). Una columna capilar (Hewlett-Packard) HP-5 (5\% fenil metil siloxano) de $60 \mathrm{~m}$ $\times 0,25 \mathrm{~mm}$ de diámetro interno $\times 0,25$ $\mu \mathrm{m}$ de espesor.

El gas portador empleado fue Helio $(99,995 \%$, Agafano) a un flujo de $2 \mathrm{~mL} / \mathrm{min}$, la temperatura del inyector a $270^{\circ} \mathrm{C}$, inyección modo splitless. Para la determinación cromatográfica se utilizó la siguiente programación del horno: $200^{\circ} \mathrm{C}$ durante dos minutos seguidamente aumento a $20^{\circ} \mathrm{C} / \mathrm{min}$ hasta $270^{\circ} \mathrm{C}$ y de ahí aumento a $3^{\circ} \mathrm{C} / \mathrm{min}$ hasta $273^{\circ} \mathrm{C}$ en donde se mantuvo constante por un minuto, luego se llevó hasta $278^{\circ} \mathrm{C}$ a $0,5^{\circ} \mathrm{C} /$ minuto y por ultimo a $10^{\circ} \mathrm{C} /$ minuto hasta alcanzar una temperatura final de $290^{\circ} \mathrm{C}$, en la cual se dejó por 4 minutos.

\section{Evaluación del material de almacenamiento:}

Se tomaron $320 \mathrm{~g}$ de cada material vegetal y se doparon con cipermetrina, posteriormente se repartieron en cantidades iguales entre cuatro recipientes diferentes (Tabla 1) y se almacenaron a temperatura ambiente.

Tabla 1. Recipientes de almacenamiento

\begin{tabular}{|c|c|}
\hline MUESTRA & $\begin{array}{c}\text { RECIPIENTES DE } \\
\text { ALMACENAMIENTO }\end{array}$ \\
\hline 1 & Recipiente de vidrio tapa rosca \\
\hline 2 & $\begin{array}{c}\text { Bolsa de polietileno de alta } \\
\text { densidad }\end{array}$ \\
\hline 3 & $\begin{array}{c}\text { Bolsa de polietileno de baja } \\
\text { densidad }\end{array}$ \\
\hline 4 & Bolsa de aluminio. \\
\hline
\end{tabular}

La determinación de la cantidad de pesticida presente en el pasto se tomó durante una semana, cada 24 horas desde el dopaje de la muestra, todos los análisis se realizaron por duplicado.
Para cada análisis se tomaron 5 gramos de material vegetal y se adicionaron $30 \mathrm{~mL}$ de una solución $1 \%$ $\mathrm{V} / \mathrm{V}$ cipermetrina en acetona, luego se dejaron $10 \mathrm{~min}$ a $60^{\circ} \mathrm{C}$ y a este tiempo se tomó una alícuota de $3 \mathrm{~mL}$ de la cual se extrajo la cipermetrina contenida por microextracción en fase sólida, exponiendo la fibra durante 30 minutos en un baño de agua termostáticamente controlado a $70^{\circ} \mathrm{C}$ y una velocidad de agitación constante de 500rpm. Una vez realizada la extracción se llevó a cabo la determinación del pesticida piretroide utilizando cromatografía de gases con detector de ionización de llama (GC-FID) bajo las condiciones expuestas anteriormente.

\section{Evaluación de las condiciones de almacenamiento:}

Después de obtener los resultados del tipo de recipiente más adecuado para el almacenamiento de la muestra, se tomaron 640 gramos de la muestra vegetal y se dividieron en 8 , utilizando el recipiente apropiado para el almacenamiento, estas muestras se doparon con $30 \mathrm{~mL}$ de una solución de cipermetrina a $13 \mathrm{ppm}$, seguidamente se almacenaron en diferentes condiciones (tabla 2). Todos los análisis se llevaron a cabo por duplicado y se analizaron siguiendo el protocolo expuesto anteriormente.

Tabla 2. Condiciones de almacenamiento 
33

\begin{tabular}{|c|c|}
\hline CONDICIÓN & DETALLE \\
\hline 1 & $\begin{array}{c}\text { Temperatura Ambiente (promedio } \\
\left.16^{\circ} \mathrm{C}\right), \text { con exposición a la luz } \\
\text { natural }\end{array}$ \\
\hline 2 & $\begin{array}{c}\text { Temperatura Ambiente (promedio } \\
\left.16^{\circ} \mathrm{C}\right) \text { sin exposición a la luz natural }\end{array}$ \\
\hline 3 & $\begin{array}{c}\text { En la nevera }\left(5^{\circ} \mathrm{C}\right), \text { sin protección } \\
\text { de la luz artificial }\end{array}$ \\
\hline 4 & $\begin{array}{c}\text { En la nevera }\left(5^{\circ} \mathrm{C}\right), \text { protegido de la } \\
\text { luz artificial con papel aluminio }\end{array}$ \\
\hline 5 & $\begin{array}{c}\text { En el horno }\left(40^{\circ} \mathrm{C}\right), \text { sin protección } \\
\text { de la luz artificial }\end{array}$ \\
\hline 6 & $\begin{array}{c}\text { En el horno }\left(40^{\circ} \mathrm{C}\right), \text { protegida de la } \\
\text { luz artificial con papel aluminio } \\
\left.\text { En el congelador (- }{ }^{\circ} \mathrm{C}\right), \text { sin } \\
\text { protección de la luz artificial }\end{array}$ \\
\hline 8 & $\begin{array}{c}\left.\text { En el congelador (- } 4^{\circ} \mathrm{C}\right), \text { protegida } \\
\text { de la luz artificial con papel } \\
\text { aluminio }\end{array}$ \\
\hline
\end{tabular}

\section{RESULTADOS Y DISCUSIÓN}

No se encontraron diferencias significativas en los resultados obtenidos para los diferentes parámetros evaluados, en las tres variedades de pasto estudiadas, posiblemente debido a la morfoanatomía y fisiología de estas pasturas, pertenecientes a la familia poaceae, que se caracteriza por tener fotosíntesis tipo C4 y compartimentos en las láminas foliares de los haces vasculares, guardan la misma distribución en los parénquimas y las esclerénquimas con un mesófilo esponjoso igualmente ordenado, por lo que este tipo de pesticida se acumula y se elimina de forma poco diferencial entre los tres tejidos ${ }^{13}$.

Teniendo en cuenta que los resultados encontrados fueron similares para las variedades de pasturas tropicales evaluadas y que el pasto Brachiaria decumbens es el más prevalente en las praderas de ganado, se dan a conocer los resultados obtenidos para esta variedad. Los resultados obtenidos en la selección del recipiente adecuado para el almacenamiento de la muestra vegetal, se encuentran en la figura 1.

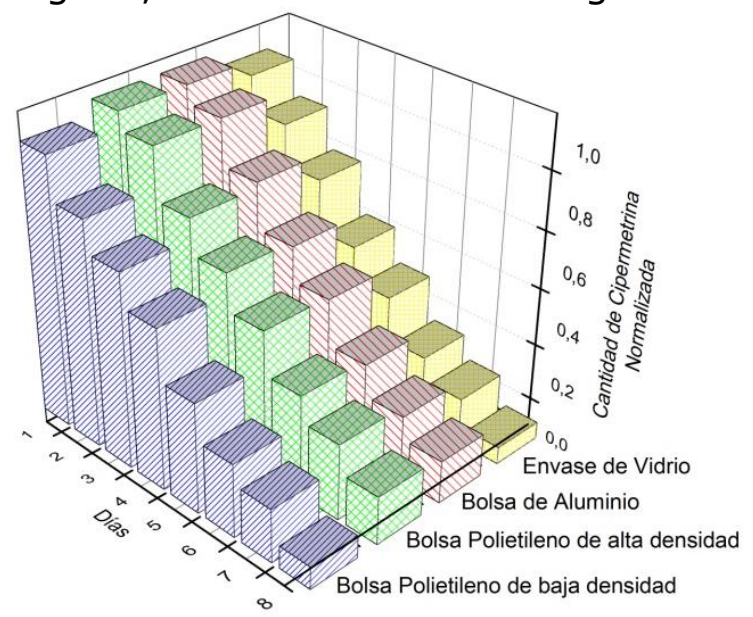

Figura 1. Comparación de los diferentes recipientes utilizados, para el almacenamiento de pasto Brachiaria decumbens a temperatura ambiente $\left(16^{\circ} \mathrm{C}\right)$.

Como se puede observar en la figura 1 , la degradación de la cipermetrina es menor al utilizar bolsa de polietileno de alta densidad o bolsa de aluminio como material de almacenamiento; sin embargo, desde las 48 horas de medición se observó que la velocidad de degradación del piretroide en las muestras que se encontraban en bolsas de polietileno alta densidad correspondía a la mitad de la velocidad de degradación que se observó en las muestras almacenadas en bolsas de aluminio.

Al realizar ANOVA (Origin, versión de prueba) a los datos obtenidos, se observó que hay diferencias estadísticamente significativas entre las bolsas de alta densidad y aluminio con respecto a los otros materiales ( $P$ valor 0,022 ); sin embargo, como se deseaba evaluar la importancia de la luz en las condiciones favorables de almacenamiento, se decidió trabajar con bolsas plásticas de alta densidad como material de almacenamiento en este estudio.

Los resultados de la evaluación de las diferentes condiciones de almacenamiento estudiadas, se dan a conocer en las Figuras 2 y 3 . Como se 
puede observar en dichas figuras la velocidad de degradación del pesticida es diferente en cada caso, lo que significa que la temperatura y la luz interfieren en la degradación del plaguicida.

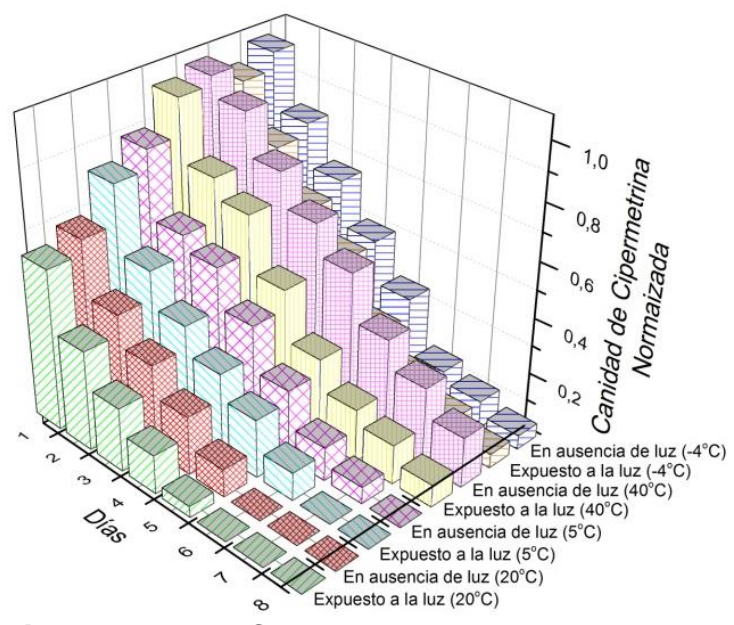

Figura 2. Cantidad Normalizada de cipermetrina en los diferentes días a las condiciones de almacenamiento estudiadas.

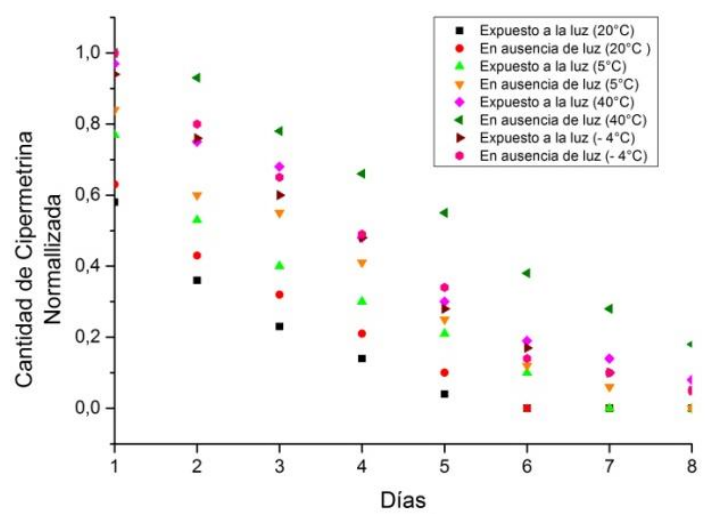

Figura 3. Degradación de la cipermetrina a las diferentes condiciones de almacenamiento estudiadas.

Se demostró una cinética de primer orden (Ver figuras 3 y 4 ) trazando el logaritmo neperiano (In) de la concentración del pesticida piretroide cipermetrina contra el tiempo de almacenamiento en las diferentes condiciones. Los coeficientes de correlación, las constantes de proporción de primer orden (Velocidad específica) y la vida media $\left(t_{1 / 2}\right)$ para las diferentes muestras se dan a conocer en la tabla 3.
Tabla 3. Parámetros cinéticos

\begin{tabular}{|c|c|c|c|}
\hline Almacenamiento & $\mathbf{R}^{2}$ & $\mathbf{K}$ & $\mathbf{t}_{1 / 2}$ (min) \\
\hline $\begin{array}{c}\text { Temperatura } \\
\text { Ambiente (promedio } \\
\left.16^{\circ} \mathrm{C}\right) \text {, con } \\
\text { exposición a la luz } \\
\text { natural. }\end{array}$ & 0,993 & 0,417 & 4612,490 \\
\hline $\begin{array}{c}\text { Temperatura } \\
\text { Ambiente (promedio } \\
\left.16^{\circ} \mathrm{C}\right) \text { sin } \\
\text { exposición a la luz } \\
\text { natural. }\end{array}$ & 0,986 & 0,403 & 5111,136 \\
\hline $\begin{array}{c}\text { En la nevera (5ㅇ), } \\
\text { sin protección de la } \\
\text { luz artificial. }\end{array}$ & 0,987 & 0,37 & 6582,672 \\
\hline $\begin{array}{c}\text { En la nevera }\left(5^{\circ} \mathrm{C}\right), \\
\text { protegido de la luz } \\
\text { artificial con papel } \\
\text { aluminio. }\end{array}$ & 0,986 & 0,313 & 6699,456 \\
\hline $\begin{array}{c}\left.\text { En el horno (40 }{ }^{\circ} \mathrm{C}\right), \\
\text { sin protección de la } \\
\text { luz artificial. . }\end{array}$ & 0,990 & 0,517 & 4186,800 \\
\hline $\begin{array}{c}\left.\text { En el horno (40 }{ }^{\circ} \mathrm{C}\right), \\
\text { protegida de la luz } \\
\text { artificial con papel } \\
\text { aluminio. }\end{array}$ & 0,993 & 0,460 & 4532,544 \\
\hline $\begin{array}{c}\text { En el congelador }(- \\
\left.4^{\circ} \mathrm{C}\right), \text { sin protección } \\
\text { de la luz artificial. }\end{array}$ & 0,972 & 0,382 & 5711,328 \\
\hline $\begin{array}{c}\text { En el congelador }(- \\
\left.4^{\circ} \mathrm{C}\right), \text { protegida de } \\
\text { la luz artificial con } \\
\text { papel aluminio. }\end{array}$ & 0,961 & 0,383 & 5710,752 \\
\hline
\end{tabular}

De acuerdo con los datos mostrados en la tabla 3, el orden creciente de estabilidad de la cipermetrina está dado, en la siguiente secuencia: Nevera $\left(5^{\circ} \mathrm{C}\right)>$ congelador $\left(-4^{\circ} \mathrm{C}\right)>$ temperatura ambiente (promedio $\left.16^{\circ} \mathrm{C}\right)>$ horno $\left(40^{\circ} \mathrm{C}\right)$. Para todos los casos, se encontró mayor estabilidad en ausencia de luz.

La cantidad de piretroide en las muestras almacenadas a $40^{\circ} \mathrm{C}$ solo pudo ser determinada durante 5 días, debido a la pérdida de peso (humedad) en el pasto. 


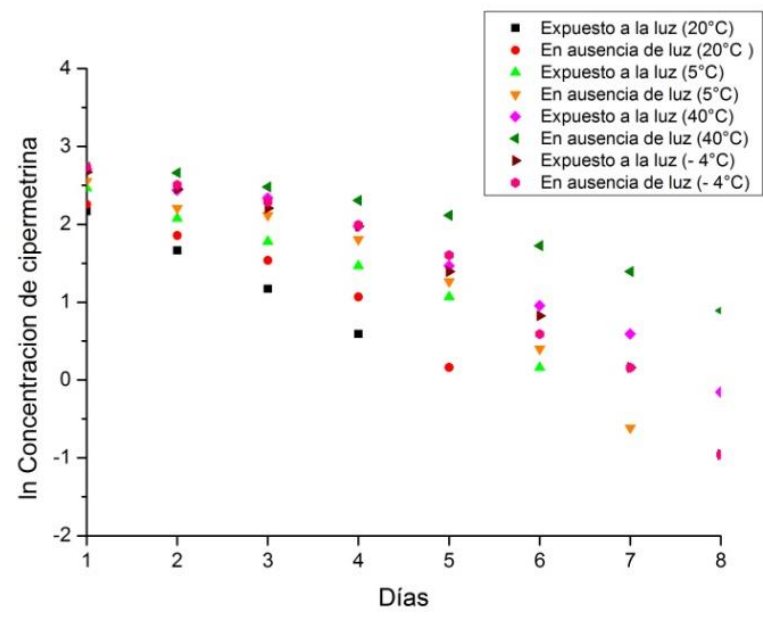

Figura 4. Logaritmo neperiano de la concentración de cipermetrina Vs tiempo de análisis.

Al realizar ANOVA y prueba de rangos múltiples (Origin, versión de prueba) a los datos obtenidos, se observó que las diferencias para ensayos en presencia o ausencia de luz no son estadísticamente significativas, pero se encontró que si existe diferencia estadísticamente significativa entre las muestras almacenadas a $5^{\circ} \mathrm{C}$ y $4^{\circ} \mathrm{C}$ con respecto a las otras con un $P$ valor de 0,031 . Además se encontró que en las muestras a 5 y $-4^{\circ} \mathrm{C}$ la diferencia de concentración del pesticida se hace significativa desde el día 6.

\section{CONCLUSIONES}

De acuerdo con los datos de las constantes de primer orden y vidas medias obtenidas, la cipermetrina es más estable a $5^{\circ} \mathrm{C}$ y $-4^{\circ} \mathrm{C}$ que a 40 o $16^{\circ} \mathrm{C}$. Si se desea almacenar un número considerable de muestras de pasturas tropicales pertenecientes a las poaceas, tratadas con cipermetrina, se recomienda almacenarlas en bolsas de polietileno de alta densidad o aluminio en una nevera a 5 o $\quad-4^{\circ} \mathrm{C}$. Durante las primeras 192 horas de almacenamiento, la exposición a la luz no es un factor importante. Las muestras se pueden almacenar bajo las condiciones recomendadas durante 5 días.

\section{AGRADECIMIENTOS}

A la Universidad de Pamplona, al laboratorio de Control de calidad y al Ministerio de Agricultura que permitió realizar está investigación dentro del proyecto según convenio MDR 2008 H24683968.

\section{Referencias bibliográficas}

1. Fortin $\mathrm{M}-\mathrm{C}$, Bouchard M, Carrier G, Dumas $P$. Biological monitoring of exposure to pyrethrins and pyrethroids in a metropolitan population of the Province of Quebec, Canada. Environmental Research. 2008;107(3):343-50.

2. Jiménez Venegas $L$, Quilodrán Peredo J, Miranda Olivares JP, Rodríguez Bustos $H$. Efecto de Dosis Única Intraperitoneal de Cipermetrina en la Corteza Cerebral Somatosensorial de Ratones CF- 1. International Journal of Morphology. 2008;26:19-26.

3. Barbini D, Vanni F, Girolimetti S, Dommarco R. Development of an analytical method for the determination of the residues of four pyrethroids in meat by GC-ECD and confirmation by GC-MS. Anal Bioanal Chem. 2007;389(6):1791-8.

4. Leng G, Gries W, Selim S. Biomarker of pyrethrum exposure. Toxicol Lett. 2006;162(2-3):195-201.

5. Niazi A, Goodarzi M. Orthogonal signal correction-partial least squares method for simultaneous spectrophotometric determination of cypermethrin and tetramethrin. Spectrochimica acta Part A, Molecular and biomolecular spectroscopy. 2008;69(4):1165-9.

6. Herrera M, Márquez M, Sierra G. Absorción y distribución de la cipermetrina en kikuyo (Pennisetum clandestinum Hochst ex chiov.) en cultivo hidropónico [Maestría]. Medellín, 
Colombia.: Universidad de Antioquia; 2009.

7. Bonansea RI, Amé MV, Wunderlin $D A$. Determination of priority pesticides in water samples combining SPE and SPME coupled to GC-MS. A case study: Suquía River basin (Argentina). Chemosphere. 2013;90(6):1860-9.

8. Fernández-Álvarez $M$, SánchezPrado L, Lores M, Llompart M, GarcíaJares C, Cela R. Alternative sample preparation method for photochemical studies based on solid phase microextraction: Synthetic pyrethroid photochemistry. Journal of Chromatography A. 2007;1152(12):156-67.

9. Filho AM, dos Santos FN, Pereira PAdP. Development, validation and application of a method based on DISPME and GC-MS for determination of pesticides of different chemical groups in surface and groundwater samples. Microchemical Journal. 2010;96(1):13945.

10. Tankiewicz M, Morrison C, Biziuk M. Multi-residue method for the determination of 16 recently used pesticides from various chemical groups in aqueous samples by using DI-SPME coupled with GC-MS. Talanta. 2013;107:1-10.

11. Pappas C, Kyriakidis NV, Athanasopoulos PE. Effects of storage conditions and fruit processing on the degradation of parathion methyl on apples and lemons. Food additives and contaminants. $2003 ; 20(4): 375-9$.

12. Universidad Nacional de La Plata y Centro de Estudios de Aguas y Suelos de Uso Agropecuario. Impacto de pesticidas en aguas superficiales y sedimentos asociado a cultivos por siembra directa. Conferencia internacional usos múltiples del agua: Para la vida y el Desarrollo Sostenible. 2003.

13. Pelaez MJ., Bustamante JJ, Chaparro AL, Calderon LS, Cardona Y, Garcia CH (2011) Efectos deletéreos de sustancias xenobióticas en la calidad de la canal y vísceras de los bovinos en algunas localidades del Magdalena Medio y Norte de Santander. Informe parcial de investigación. Proyecto Ministerio de Desarrollo Económico y RuralUniversidad de Pamplona, Republica de Colombia. 2011.

Yaneth Cardona.MsC.Departamento de Química. Facultad de Ciencias Basicas. Universidad de Pamplona.

Manuel Peláez.Ph.D. Docente Ciencias Biologicas .Universidad Nacional.PalmiraValle del cauca.Universidad Nacional

Amanda Chaparro.Ph.D. Profesor Asociado.Departamento Química.Facultad de de Basicas Universidad Colombia 
